Research Paper

\title{
Pooling-analysis on hMLH1 polymorphisms and cancer risk: evidence based on 31,484 cancer cases and 45,494 cancer-free controls
}

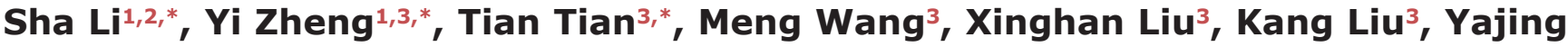 \\ Zhai $^{1}$, Cong Dai ${ }^{3}$, Yujiao Deng ${ }^{3}$, Shanli $\mathrm{Li}^{3}$, Zhijun Dai ${ }^{3}$ and Jun Lu ${ }^{1}$ \\ ${ }^{1}$ Clinical Research Center, First Affiliated Hospital of Xi'an Jiaotong University, Xi'an, Shaanxi 710061, China \\ ${ }^{2}$ Department of Pharmacy, Second Affiliated Hospital of Xi'an Jiaotong University, Xi'an, Shaanxi 710004, China \\ ${ }^{3}$ Department of Oncology, Second Affiliated Hospital of Xi'an Jiaotong University, Xi'an, Shaanxi 710004, China \\ *These authors have contributed equally to this work \\ Correspondence to: Jun Lu, email: lujun2006@xjtu.edu.cn \\ Zhijun Dai, email: dzj0911@126.com
}

Keywords: $h M L H 1$, polymorphism, cancer, meta-analysis

Received: May 23, $2017 \quad$ Accepted: September 08, $2017 \quad$ Published: October 10, 2017

Copyright: Li et al. This is an open-access article distributed under the terms of the Creative Commons Attribution License 3.0 (CC BY 3.0), which permits unrestricted use, distribution, and reproduction in any medium, provided the original author and source are credited.

\section{ABSTRACT}

To elucidate the veritable relationship between three hMLH1 polymorphisms (rs1800734, rs1799977, rs63750447) and cancer risk, we performed this metaanalysis based on overall published data up to May 2017, from PubMed, Web of knowledge, VIP, WanFang and CNKI database, and the references of the original studies or review articles. 57 publications including 31,484 cancer cases and 45,494 cancer-free controls were obtained. The quality assessment of six articles obtained a summarized score less than 6 in terms of the Newcastle-Ottawa Scale (NOS). All statistical analyses were calculated with the software STATA (Version 14.0; Stata Corp, College Station, TX). We found all the three polymorphisms can enhance overall cancer risk, especially in Asians, under different genetic comparisons. In the subgroup analysis by cancer type, we found a moderate association between rs1800734 and the risk of gastric cancer (allele model: $O R=1.14, P=0.017$; homozygote model: $O R=1.33, P=0.019$; dominant model: $O R=1.27, P=0.024)$ and lung cancer in recessive model $(O R=1.27, P=0.024)$. The $G$ allele of rs1799977 polymorphism was proved to connect with susceptibility of colorectal cancer (allele model: $O R=1.21$, $P=0.023$; dominate model: $O R=1.32, P<0.0001$ ) and prostate cancer (dominate model: $O R=1.36, P<0.0001)$. Rs63750447 showed an increased risk of colorectal cancer, endometrial cancer and gastric cancer under all genetic models. These findings provide evidence that hMLH1 polymorphisms may associate with cancer risk, especially in Asians.

\section{INTRODUCTION}

As one of the pivotal pathways in maintaining genetic stability, MMR system is mainly in charge of repairing the replication-associated errors, including removing mistaken bases, correcting substitutions and rectifying insertion-deletion mismatches. Its defects may result in microsatellite instability (MSI), a type of genetic instability related to colorectal cancer, gastric cancer, and endometrial cancer, etc. [1-3] Interest in MLH1 has increased in the last few years because MLH1 was discovered as a key component in MMR for MSI, and its dysfunction is supposed to be implicated in cancer predisposition.

MLH1 not only takes part in the activities of MMR system, but also has other interesting cellular functions, such as participating in cell cycle arrest, triggering DNA damage-induced apoptosis to response to some chemical 
or physical agents [4], and interacting with tumorrelated signaling molecules like BRCA1 [5] and p53 [6]. Moreover, various polymorphisms were found in MLH1 gene, part of them were proved to influence the expression of functional MLH1. We selected three most common loci rs 1800734, rs1799977, and rs63750447 in hMLH1 which may alter the function of the hMLH1 gene according to literature. Among these, the A allele of rs 1800734 polymorphism could alter the methylation level of nine CpG sites mapped on the MLH1 promoter [7], while rs1799977 and rs63750447 were situated at the exons of hMLH1 [1, 8]. Emerging inspiring evidences indicate these functional polymorphisms of hMLH1 may be potential candidates in mediating hereditary susceptibility to cancer, however, applying them in clinical application is still treated critically. Past decades witnessed numerous molecular epidemiological studies carried out worldwide to investigate the actual association between them, yet no coincident conclusion was reached so far.

For example, Nizam et al. [9] concluded that rs1800734 polymorphism had an influence on colorectal cancer (CRC) risk among Malaysians in 2013, while Zhang et al [10] found no obvious connection between rs 1800734 and CRC risk in 2016. For rs1799977 polymorphism, Milanizadeh et al. [11] detected it could increase CRC risk particularly in female patients, but Peng et al. [1] hold a contrary opinion that no association existed between the two. The inconsistent conclusions also existed in the studies exploring the relationship between hMLH1 polymorphisms and other cancer types. Although rs63750447 polymorphism was accepted as a risk factor for east-Asian CRC patients [1, 12-14], no reliable conclusion reported on the possible relationship between rs63750447 and overall cancer or other kinds of tumors. To solved these controversies, a comprehensive and persuasive meta-analysis was excepted to conduct depending on complete published data and proper methodological tools, thus we carried out this metaanalysis to illuminate the objective connection between hMLH1 polymorphisms (rs1800734, rs1799977 and rs63750447) and cancer risk.

\section{RESULTS}

\section{Characteristics of eligible studies}

Finally, we obtained a total of 57 publications including 31,484 cancer cases and 45,494 cancer-free controls (all were from the databases and no study was identified by manual search of the references of the original studies or review articles). The detail selection process was shown in the flow diagram (Figure 1). What needed illustration is that we abandon three studies contained in previous meta-analyses after comprehensive reading full text. The first one was the study performed by Chen et al [15], contained in the meta-analyses conducted in 2011 [16] and 2015 [17], which was excluded on account of both its cases group and controls group are women with cancers (cases with MLH1 methylation while controls not). Another study finished by van Roon et al. [18], also included in previous meta-analyses [17, 19], has two controls groups collected from literature [20,21]. We excluded it after discussing with a senior author within us. And the third study we abandoned was due to deficiency of cancer-free control group [16].

Among the 57 eligible literatures, 26 were based on Caucasian background from, Poland, Spain, the United States, Denmark, the United Kingdom, Sweden, Portugal, Czech Republic and Canada. 27 were carried out in Asians from China, Kazakhstan, India, Iran, Malaysia, Japan and Korea, and four were based on mixed ethnic groups. All the publications involving rs63750447 polymorphism were carried out among the Chinese population. Three casecohort designed studies [22-24] and 54 case-controlled studies were involved in this meta-analysis. All cancer cases were confirmed by pathology or histology, involved cancer types covering colorectal, gastric, ovarian, head and neck, endometrium, lung, bladder, prostate, thyroid, breast, prostate, Non-Hodgkin lymphoma, acute myeloid leukaemia, and acute lymphoblastic leukaemia. The quality assessment of six studies obtained a summarized score less than 6 in terms of the Newcastle-Ottawa Scale (NOS), four of them are studying on rs 1800734 [25-28] while one of them is for rs63750447 [29], and the other one focused on rs1800734 and rs1799977 polymorphisms [30]. Specially, two publications by Zhang et al. [8] and Wang et al. [29] contained four and three independent studies respectively. One study focused on rs1799977 polymorphism by Joshi et al. [31] did not provide complete genotype frequencies. Hence only the dominant model was evaluated. Detail characteristics of eligible publications are displayed in Table 1.

\section{Quantitative synthesis}

The distributions of genotypes frequencies of hMLH1 polymorphisms (rs1800734; rs1799977; rs63750447) for every single study are exhibited in Table 2. The minor allele frequencies (MAF) among cancer cases varied widely according to the included studies, ranging from 0.205 to 0.656 for rs 1800734 polymorphism, 0.016 to 0.744 for rs 1799977 polymorphism, and 0.032 to 0.069 for rs63750447 polymorphism. The average MAF of case-group for the three polymorphisms is 0.396 , $0.233,0.053$, respectively. The meta-analysis results of these three polymorphisms were shown in Supplementary Table 1.

\section{Rs1800734 polymorphism}

Overall, there are 39 studies including 29,331 cases and 29,588 controls for rs 1800734 polymorphism. Statistically significance was found between rs 1800734 polymorphism and overall cancer risk under five genetic 
models (recessive comparison: $\mathrm{OR}=1.22,95 \% \mathrm{CI}=1.09$ $1.37, P=0.001$; homozygote comparison: $\mathrm{OR}=1.23$, $95 \% \mathrm{CI}=1.06-1.42, P=0.006$; allele comparison: OR $=1.08,95 \% \mathrm{CI}=1.01-1.16, P=0.023)$. After excluding nine studies that were not in accordance with $\operatorname{HWE}[3,9$, $25,26,32-36]$, we observed increased risks of all kinds of cancers under two genetic models (recessive comparison: $\mathrm{OR}=1.18,95 \% \mathrm{CI}=1.04-1.34, P=0.012$; homozygote comparison: $\mathrm{OR}=1.18,95 \% \mathrm{CI}=1.00-1.39, \mathrm{P}=0.048$, Figure 2A).

In the stratification analysis based on ethnicity (Figure 3A), we found no association between cancer risk and Caucasian population, while the mutation allele
A contributed to an increasing cancer risk in Asian population under three comparison models (recessive comparison: $\mathrm{OR}=1.30,95 \% \mathrm{CI}=1.11-1.53, P=0.001$; homozygote comparison: $\mathrm{OR}=1.37,95 \% \mathrm{CI}=1.09-1.72$, $P=0.006$; allele comparison: $\mathrm{OR}=1.16,95 \% \mathrm{CI}=1.03$ $1.31, P=0.014)$. In the cancer-specific analysis, rs 1800734 polymorphism showed a potential tendency to enhance gastric and lung cancer susceptibility in different genetic comparisons (gastric cancer: dominate comparison: OR $=1.27,95 \% \mathrm{CI}=1.03-1.56, P=0.024$; homozygote comparison: $\mathrm{OR}=1.33,95 \% \mathrm{CI}=1.06-1.68, P=0.019$, allele comparison: $\mathrm{OR}=1.14,95 \% \mathrm{CI}=1.02-1.28, P=$ 0.017 ; lung cancer: recessive comparison: $\mathrm{OR}=1.27$,

\section{PRISMA 2009 Flow Diagra}

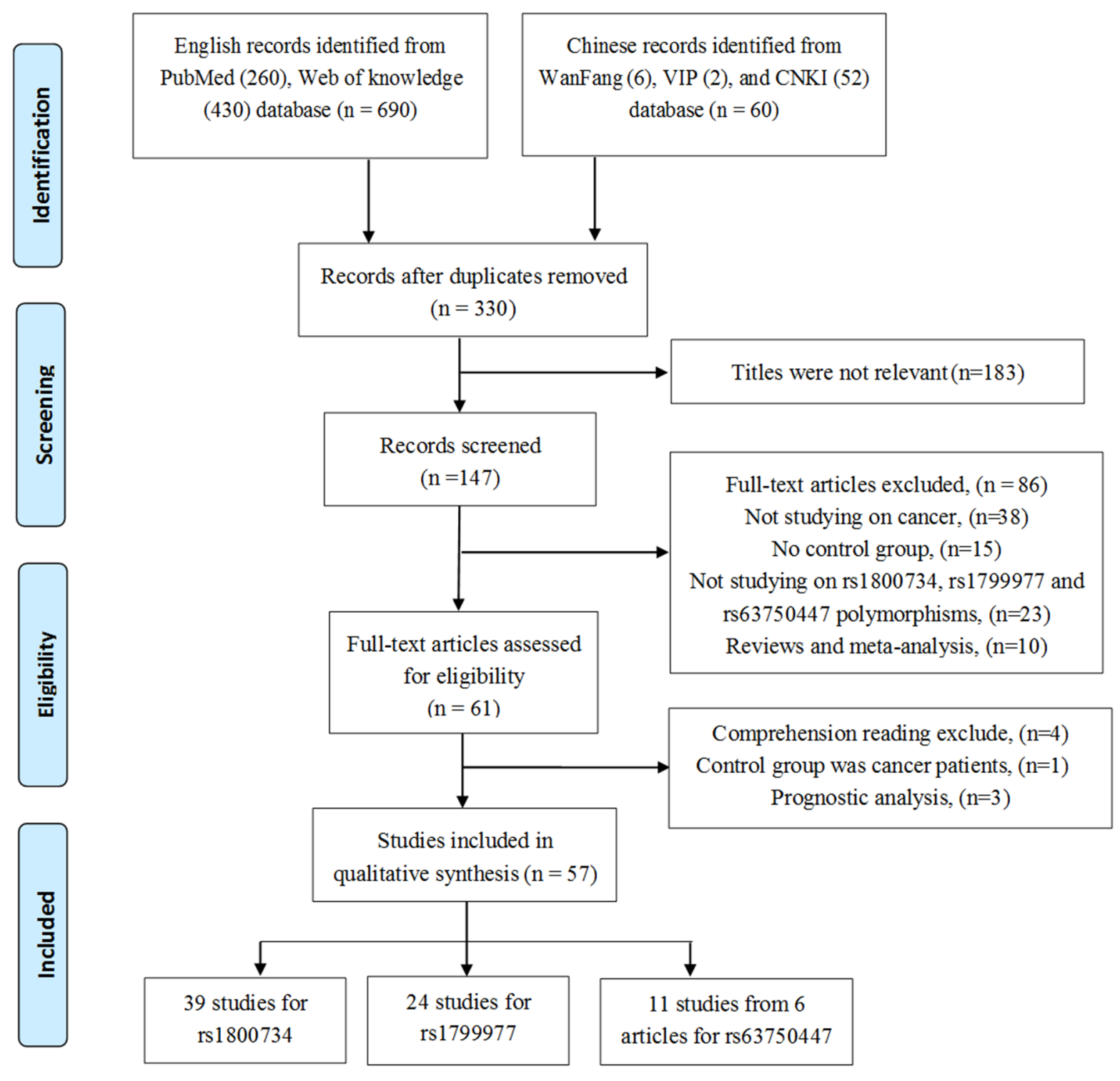

Figure 1: The flow diagram of the meta-analysis, according to the PRISMA 2009. CNKI = China National Knowledge Infrastructure. 
Table 1: Characteristics of the studies included in the meta-analysis

\begin{tabular}{|c|c|c|c|c|c|c|c|c|}
\hline First author & Year & Country & Ethnic & Method & Control & Disease & SNP & NOS \\
\hline Peng [1] & 2016 & China & Asian & PCR-HRM & Population & $\mathrm{CRC}$ & 2,3 & 7 \\
\hline Zhang [10] & 2016 & China & Asian & TaqMan & Hospital & $\mathrm{CRC}$ & 1 & 6 \\
\hline Zhu [2] & 2016 & China & Asian & TaqMan & Population & $\mathrm{GC}$ & 1 & 7 \\
\hline Djansugurova [46] & 2015 & Kazakhstan & Asian & PCR-RFLP & Hospital & $\mathrm{CRC}$ & 1 & 8 \\
\hline Niu [47] & 2015 & China & Asian & PCR-RFLP & Hospital & $\mathrm{OC}$ & 1,2 & 6 \\
\hline Nogueira [48] & 2015 & Brazil & Mixed & TaqMan & Hospital & HNSCC & 1 & 6 \\
\hline Poplawski [3] & 2015 & Poland & Caucasian & PCR-RFLP & Hospital & $\mathrm{EC}$ & 1 & 6 \\
\hline Slovakova [49] & 2015 & Slovak & Caucasian & PCR-RFLP & Population & $\mathrm{LC}$ & 1 & 8 \\
\hline Rodriguez [50] & 2014 & Spain & Caucasian & PCR-RFLP & Hospital & BT & 1 & 6 \\
\hline Jha [51] & 2013 & India & Asian & PCR-RFLP & Population & HNSCC & 1 & 7 \\
\hline Martinez-Uruena [25] & 2013 & Spain & Caucasian & PCR-RFLP & Hosptal & $\mathrm{CRC}$ & 1 & 4 \\
\hline Milanizadeh [11] & 2013 & Iran & Asian & PCR-RFLP & Hospital & $\mathrm{CRC}$ & 2 & 7 \\
\hline Nizam [9] & 2013 & Malaysia & Asian & PCR-RFLP & Hospital & $\mathrm{CRC}$ & 1 & 6 \\
\hline Muniz-Mendoza [30] & 2012 & Mexico & Mixed & PCR-RFLP & Hospital & $\mathrm{CRC}$ & 1,2 & 4 \\
\hline Savio [32] & 2012 & Canada & Caucasian & PCR-RFLP & Population & $\mathrm{CRC}$ & 1 & 7 \\
\hline Xiao [52] & 2012 & China & Asian & PCR & Population & $\mathrm{GC}$ & 1,2 & 8 \\
\hline Zhi [53] & 2012 & China & Asian & PCR-RFLP & Population & BLC & 1 & 7 \\
\hline Lacey [54] & 2011 & Poland & Caucasian & iSelect bead chip & Population & $\mathrm{EC}$ & 1,2 & 8 \\
\hline Lo $[55]$ & 2011 & China & Asian & PCR & Hospital & $\mathrm{LC}$ & 1 & 7 \\
\hline Soni $[56]$ & 2011 & India & Asian & TaqMan & Hospital & $\mathrm{PC}$ & 1 & 6 \\
\hline Whiffin [57] & 2011 & UK & Asian & KASPae & Population & $\mathrm{CRC}$ & 1 & 8 \\
\hline Zhi [58] & 2011 & China & Asian & PCR-RHM & Hospital & $\mathrm{GC}$ & 1 & 6 \\
\hline Langeberg [59] & 2010 & USA & Caucasian & ABI & Population & $\mathrm{PC}$ & 2 & 7 \\
\hline Picelli [22] & 2010 & Sweden & Caucasian & Direct sequencing & Population & $\mathrm{CRC}$ & 2 & 7 \\
\hline Shi [12] & 2010 & China & Asian & PCR & Hospital & $\mathrm{TC}$ & $1,2,3$ & 6 \\
\hline Campbell [41] & 2009 & USA & Caucasian & PCR-RFLP & Population & $\mathrm{CRC}$ & 1,2 & 8 \\
\hline Conde [37] & 2009 & Portugal & Caucasian & QIAamp & Hospital & $\mathrm{BC}$ & 2 & 6 \\
\hline Joshi [31] & 2009 & USA & Caucasian & TaqMan & Population & $\mathrm{CRC}$ & 2 & 7 \\
\hline Nejda [38] & 2009 & Spain & Caucasian & PCR-RFLP & Hospital & $\mathrm{CRC}$ & 2 & 7 \\
\hline Ohsawa [13] & 2009 & Japan & Asian & PCR-RFLP & Unknown & $\mathrm{CRC}$ & 3 & 6 \\
\hline Shih [33] & 2009 & China & Asian & PCR-RFLP & Population & $\mathrm{LC}$ & 1 & 7 \\
\hline Tanaka [60] & 2009 & Japan & Asian & Direct sequencing & Population & $\mathrm{PC}$ & 2 & 7 \\
\hline An [61] & 2008 & China & Asian & PCR-RFLP & Population & $\mathrm{LC}$ & 1,2 & 8 \\
\hline Christensen [23] & 2008 & Denmark & Caucasian & SBE-tags & Population & $\mathrm{CRC}$ & 2 & 8 \\
\hline Harlay [26] & 2008 & Canada & Mixed & MassARRAY & Hospital & $\mathrm{OC}$ & 1 & 5 \\
\hline Koessler [62] & 2008 & UK & Caucasian & TaqMan & Population & $\mathrm{CRC}$ & 1 & 7 \\
\hline Samowitz [20] & 2008 & USA & Caucasian & Direct sequencing & Population & $\mathrm{CRC}$ & $\begin{array}{l}1 \\
(C o\end{array}$ & $\begin{array}{c}7 \\
\text { nued) }\end{array}$ \\
\hline
\end{tabular}




\begin{tabular}{|c|c|c|c|c|c|c|c|c|}
\hline First author & Year & Country & Ethnic & Method & Control & Disease & SNP & NOS \\
\hline Scott [34] & 2008 & UK & Caucasian & TaqMan & Population & NHL & 1 & 6 \\
\hline Tulupova [63] & 2008 & Czech & Caucasian & TaqMan & Hospital & $\mathrm{CRC}$ & 1 & 7 \\
\hline Worrillow [64] & 2008 & UK & Caucasian & PCR-RFLP & Population & AML & 1 & 6 \\
\hline Berndt [24] & 2007 & USA & Caucasian & TaqMan & Population & $\mathrm{CRC}$ & 2 & 8 \\
\hline Raptis [21] & 2007 & Canada & Caucasian & TaqMan & Population & $\mathrm{CRC}$ & 1,2 & 7 \\
\hline Beiner [35] & 2006 & Canada & Mixed & MassARRAY & Hospital & $\mathrm{EC}$ & 1 & 6 \\
\hline Landi [65] & 2006 & Mixed & Caucasian & PCR & Hospital & LC & 2 & 7 \\
\hline Mei [14] & 2006 & China & Asian & PCR & Hospital & $\mathrm{CRC}$ & 2,3 & 6 \\
\hline Song [39] & 2006 & Mixed & Caucasian & TaqMan & Population & $\mathrm{OC}$ & 1,2 & 6 \\
\hline Chen [66] & 2005 & China & Asian & PCR-RFLP & Hospital & $\mathrm{HCC}$ & 1 & 7 \\
\hline Lee [67] & 2005 & Korea & Caucasian & MassARRAY & Hospital & $\mathrm{BC}$ & 1 & 6 \\
\hline $\operatorname{Kim}[68]$ & 2004 & Korea & Asian & TaqMan & Population & $\mathrm{CRC}$ & 2 & 6 \\
\hline Listgarten [40] & 2004 & Canada & Caucasian & QIAmp & Hospital & $\mathrm{BC}$ & 2 & 6 \\
\hline Park [36] & 2004 & Korea & Caucasian & PCR & Population & $\mathrm{LC}$ & 1 & 8 \\
\hline Zhang [8] & 2004 & China & Asian & DHPLC & Population & Mixed & 3 & 7 \\
\hline Deng [69] & 2003 & China & Asian & DHPLC & Hospital & GC & 1 & 7 \\
\hline Mathonnet [70] & 2003 & Canada & Caucasian & PCR-ASO & Population & ALL & 2 & 6 \\
\hline Shin [27] & 2002 & Korea & Asian & PCR-SSCP & Hospital & CRC & 1 & 4 \\
\hline Wang [29] & 2000 & China & Asian & PCR-SSCP & Hospital & Mixed & 3 & 5 \\
\hline Ito [28] & 1999 & Japan & Asian & PCR-SSCP & Hospital & $\mathrm{CRC}$ & 1 & 4 \\
\hline
\end{tabular}

$95 \% \mathrm{CI}=1.03-1.57, P=0.024)$. Besides, the subgroup analysis depended on the source of controls suggested us that rs 1800734 polymorphism had an influence on cancer risk under four genetic models among population-based controls (dominate comparison: $\mathrm{OR}=1.05,95 \% \mathrm{CI}=$ 1.01-1.10, $P=0.016$, recessive comparison: $\mathrm{OR}=1.12$, $95 \% \mathrm{CI}=1.04-1.22, P=0.004$; homozygote comparison: $\mathrm{OR}=1.22,95 \% \mathrm{CI}=1.00-1.49, P=0.050$; heterozygous comparison: $\mathrm{OR}=1.05,95 \% \mathrm{CI}=1.01-1.10, P=0.031$; allele comparison: $\mathrm{OR}=1.10,95 \%=1.00-1.20, P=$ 0.041 ) and recessive comparison among hospital-based controls $(\mathrm{OR}=1.27,95 \% \mathrm{CI}=1.03-1.57, P=0.024)$. And, when the subgroup analysis was conducted based on a quality score, rs 1800734 polymorphism displayed an increased cancer risk among high-quality studies, but no association was found among low-quality studies (Supplementary Table 1).

\section{Rs1799977 polymorphism}

We finally derived 11,665 cases and 15,538 controls from 24 eligible studies for rs 1799977 polymorphism. All the studies obtained high-quality scores according to the Newcastle-Ottawa Scale (NOS). In general, we found the variant $\mathrm{G}$ allele of rs 1799977 could improve overall cancer risks under three genetic models (dominant comparison: $\mathrm{OR}=1.28,95 \% \mathrm{CI}=1.16-1.41, P<0.0001$; homozygote comparison: $\mathrm{OR}=1.15,95 \% \mathrm{CI}=1.04-1.27, P=0.006$; allele comparison: $\mathrm{OR}=1.12,95 \% \mathrm{CI}=1.02-1.23, P=$ $0.017)$. After excluding four studies [37-40] that were not in accordance with HWE (Figure 2B), the pooled ORs and $95 \% \mathrm{CI}$ revealed a possible increased risk of cancer (dominant comparison: $\mathrm{OR}=1.25,95 \% \mathrm{CI}=1.18-1.33, P$ $<0.0001$; homozygote comparison: $\mathrm{OR}=1.13,95 \% \mathrm{CI}=$ $1.01-1.26, P=0.027$ ).

When the subgroup carried out by ethnicity (Figure 3B), a significant association was observed between rs 1799977 and cancer risk among Asians in four genetic models $($ dominant comparison: $\mathrm{OR}=1.52,95 \% \mathrm{CI}=$ 1.04-2.24, $P=0.033$; recessive comparison: $\mathrm{OR}=3.34$, $95 \% \mathrm{CI}=2.33-4.78, P<0.0001$; homozygote comparison: $\mathrm{OR}=3.44,95 \% \mathrm{CI}=2.12-5.59, P<0.0001$; allele comparison: $\mathrm{OR}=1.64,95 \% \mathrm{CI}=1.38-1.95, P<0.0001)$ and Caucasians in only dominant model $(\mathrm{OR}=1.24$, $95 \% \mathrm{CI}=1.16-1.32, P<0.0001)$. In the cancer-specific analysis (Figure 4A), rs1799977 polymorphism showed a correlation between colorectal cancer under two genetic models (dominant comparison: $\mathrm{OR}=1.32,95 \% \mathrm{CI}=1.16$ $1.51, P<0.0001$; allele comparison: $\mathrm{OR}=1.21,95 \% \mathrm{CI}=$ 
Table 2: Genotype distribution and allele frequency of hMLH1 polymorphisms

\begin{tabular}{|c|c|c|c|c|c|c|c|c|c|c|c|c|c|c|}
\hline \multirow{3}{*}{ First author } & \multicolumn{8}{|c|}{ Genotype (N) } & \multicolumn{4}{|c|}{ Allele frequency $(\mathrm{N})$} & \multirow{3}{*}{ MAF } & \multirow{3}{*}{ HWE } \\
\hline & \multicolumn{4}{|c|}{ Case (n) } & \multicolumn{4}{|c|}{ Control (n) } & \multicolumn{2}{|c|}{ Case (n) } & \multicolumn{2}{|c|}{ Control (n) } & & \\
\hline & total & $\mathbf{A A}$ & $\mathbf{A B}$ & BB & total & $\mathbf{A A}$ & $\mathbf{A B}$ & BB & $\mathbf{A}$ & B & $\mathbf{A}$ & B & & \\
\hline \multicolumn{15}{|l|}{$-93 G>A(r s 1800734)$} \\
\hline Zhang 2016 [10] & 312 & 66 & 139 & 107 & 300 & 52 & 154 & 94 & 271 & 353 & 258 & 342 & 0.566 & 0.414 \\
\hline Zhu 2016 [2] & 406 & 49 & 213 & 144 & 444 & 79 & 235 & 130 & 311 & 501 & 393 & 495 & 0.617 & 0.125 \\
\hline Niu 2015 [47] & 421 & 51 & 188 & 182 & 689 & 150 & 356 & 183 & 290 & 552 & 656 & 722 & 0.656 & 0.348 \\
\hline Djansugurova 2015 [46] & 249 & 126 & 94 & 29 & 244 & 101 & 115 & 28 & 346 & 152 & 317 & 171 & 0.305 & 0.581 \\
\hline Nogueira 2015 [48] & 450 & 248 & 171 & 31 & 450 & 269 & 159 & 22 & 667 & 233 & 697 & 203 & 0.259 & 0.809 \\
\hline Poplawski 2015 [3] & 100 & 18 & 81 & 1 & 100 & 9 & 50 & 41 & 117 & 83 & 68 & 132 & 0.415 & 0.254 \\
\hline Slovakova 2015 [49] & 422 & 250 & 144 & 28 & 511 & 260 & 228 & 23 & 644 & 200 & 748 & 274 & 0.237 & 0.002 \\
\hline Rodriguez 2014 [50] & 115 & 61 & 44 & 10 & 200 & 115 & 79 & 6 & 166 & 64 & 309 & 91 & 0.278 & 0.080 \\
\hline Jha 2013 [51] & 245 & 52 & 90 & 100 & 205 & 98 & 79 & 28 & 194 & 290 & 275 & 135 & 0.599 & 0.067 \\
\hline Martinez-Uruena2013 [25] & 383 & 233 & 131 & 19 & 236 & 129 & 102 & 5 & 597 & 169 & 360 & 112 & 0.221 & 0.003 \\
\hline Nizam 2013 [9] & 104 & 22 & 50 & 32 & 104 & 33 & 33 & 38 & 94 & 114 & 99 & 109 & 0.548 & 0.000 \\
\hline Muniz-Mendoza2012 [30] & 100 & 47 & 44 & 9 & 115 & 39 & 55 & 21 & 138 & 62 & 133 & 97 & 0.310 & 0.835 \\
\hline Savio 2012 [32] & 252 & 150 & 96 & 6 & 845 & 528 & 264 & 53 & 396 & 108 & 1320 & 370 & 0.214 & 0.012 \\
\hline Xiao 2012 [52] & 554 & 104 & 262 & 188 & 588 & 124 & 271 & 193 & 470 & 638 & 519 & 657 & 0.576 & 0.113 \\
\hline Zhi 2012 [53] & 311 & 43 & 163 & 105 & 302 & 41 & 161 & 100 & 249 & 373 & 243 & 361 & 0.600 & 0.059 \\
\hline Larcy 2011 [54] & 414 & 251 & 141 & 22 & 404 & 241 & 146 & 17 & 643 & 185 & 628 & 180 & 0.223 & 0.381 \\
\hline Lo 2011 [55] & 719 & 235 & 344 & 140 & 728 & 256 & 366 & 106 & 814 & 624 & 878 & 578 & 0.434 & 0.177 \\
\hline Soni 2011 [56] & 105 & 44 & 40 & 21 & 106 & 27 & 61 & 18 & 128 & 82 & 115 & 97 & 0.390 & 0.101 \\
\hline Whiffin 2011 [57] & 10409 & 6408 & 3504 & 497 & 6965 & 4395 & 2261 & 309 & 16320 & 4498 & 11051 & 2879 & 0.216 & 0.401 \\
\hline Zhi 2011 [58] & 236 & 36 & 111 & 89 & 240 & 42 & 114 & 84 & 183 & 289 & 198 & 282 & 0.612 & 0.757 \\
\hline Shi 2010 [12] & 204 & 40 & 102 & 62 & 204 & 34 & 99 & 71 & 182 & 226 & 167 & 241 & 0.554 & 0.959 \\
\hline Campbell 2009 [33] & 1600 & 952 & 553 & 95 & 1963 & 1170 & 688 & 105 & 2457 & 743 & 3028 & 898 & 0.232 & 0.769 \\
\hline Shih 2009 [33] & 165 & 41 & 64 & 60 & 193 & 36 & 113 & 44 & 146 & 184 & 185 & 201 & 0.558 & 0.016 \\
\hline An 2008 [61] & 500 & 163 & 243 & 94 & 517 & 169 & 258 & 90 & 569 & 431 & 596 & 438 & 0.431 & 0.618 \\
\hline Harley 2008 [26] & 842 & 483 & 297 & 62 & 776 & 532 & 206 & 38 & 1263 & 421 & 1270 & 282 & 0.250 & 0.003 \\
\hline Koessler 2008 [62] & 2288 & 1407 & 778 & 103 & 2276 & 1392 & 777 & 107 & 3592 & 984 & 3561 & 991 & 0.215 & 0.914 \\
\hline Samowitz 2008 [20] & 1006 & 610 & 344 & 52 & 1963 & 1170 & 688 & 105 & 1564 & 448 & 3028 & 898 & 0.223 & 0.769 \\
\hline Scott 2008 [34] & 601 & 375 & 205 & 21 & 942 & 610 & 310 & 22 & 955 & 247 & 1530 & 354 & 0.205 & 0.016 \\
\hline Tulupova 2008 [63] & 619 & 359 & 216 & 44 & 611 & 365 & 209 & 37 & 934 & 304 & 939 & 283 & 0.246 & 0.336 \\
\hline Worrillow 2008 [64] & 390 & 246 & 128 & 16 & 918 & 585 & 292 & 41 & 620 & 160 & 1462 & 374 & 0.205 & 0.554 \\
\hline Raptis 2007 [21] & 929 & 554 & 331 & 44 & 1098 & 687 & 352 & 59 & 1439 & 419 & 1726 & 470 & 0.226 & 0.118 \\
\hline Beiner 2006 [35] & 654 & 377 & 220 & 57 & 764 & 524 & 202 & 38 & 974 & 334 & 1250 & 278 & 0.255 & 0.002 \\
\hline Song 2006 [39] & 1306 & 825 & 414 & 67 & 1951 & 1224 & 638 & 89 & 2064 & 548 & 3086 & 816 & 0.210 & 0.615 \\
\hline Chen 2005 [66] & 545 & 86 & 261 & 198 & 374 & 85 & 178 & 111 & 433 & 657 & 348 & 400 & 0.603 & 0.400 \\
\hline Lee 2005 [67] & 783 & 201 & 348 & 234 & 594 & 117 & 292 & 185 & 750 & 816 & 526 & 662 & 0.521 & 0.927 \\
\hline Park 2004 [36] & 372 & 66 & 176 & 130 & 371 & 71 & 206 & 94 & 308 & 436 & 348 & 394 & 0.586 & 0.027 \\
\hline Deng 2003 [69] & 54 & 8 & 27 & 19 & 56 & 9 & 29 & 18 & 43 & 65 & 47 & 65 & 0.602 & 0.636 \\
\hline Shin 2002 [27] & 139 & 33 & 61 & 45 & 157 & 42 & 74 & 41 & 127 & 151 & 158 & 156 & 0.543 & 0.473 \\
\hline
\end{tabular}




\begin{tabular}{|c|c|c|c|c|c|c|c|c|c|c|c|c|c|c|}
\hline \multirow{3}{*}{ First author } & \multicolumn{8}{|c|}{ Genotype (N) } & \multicolumn{4}{|c|}{ Allele frequency $(\mathrm{N})$} & \multirow{3}{*}{ MAF } & \multirow{3}{*}{ HWE } \\
\hline & \multicolumn{4}{|c|}{ Case (n) } & \multicolumn{4}{|c|}{ Control (n) } & \multicolumn{2}{|c|}{ Case (n) } & \multicolumn{2}{|c|}{ Control (n) } & & \\
\hline & total & $\mathbf{A A}$ & $\mathbf{A B}$ & BB & total & $\mathbf{A A}$ & $\mathbf{A B}$ & BB & $\mathbf{A}$ & B & A & B & & \\
\hline Ito 1999 [28] & 27 & 8 & 10 & 9 & 84 & 22 & 46 & 16 & 26 & 28 & 90 & 78 & 0.519 & 0.355 \\
\hline \multicolumn{15}{|l|}{$655 A>G(r s 1799977)$} \\
\hline Peng2016 [1] & 156 & 151 & 5 & 0 & 311 & 307 & 4 & 0 & 307 & 5 & 618 & 4 & 0.016 & 0.909 \\
\hline Niu 2015 [47] & 418 & 383 & 33 & 2 & 689 & 613 & 75 & 1 & 799 & 37 & 1301 & 77 & 0.044 & 0.406 \\
\hline Milanizadeh 2013 [11] & 219 & 25 & 62 & 132 & 248 & 54 & 119 & 75 & 112 & 326 & 227 & 269 & 0.744 & 0.599 \\
\hline Muniz-Mendoza 2012 [30] & 102 & 71 & 26 & 5 & 100 & 81 & 19 & 0 & 168 & 36 & 181 & 19 & 0.176 & 0.294 \\
\hline Xiao 2012 [52] & 554 & 522 & 31 & 1 & 592 & 568 & 23 & 1 & 1075 & 33 & 1159 & 25 & 0.030 & 0.143 \\
\hline Larcy 2011 [54] & 417 & 210 & 160 & 47 & 406 & 196 & 165 & 45 & 580 & 254 & 557 & 255 & 0.305 & 0.253 \\
\hline Langeberg 2010 [59] & 1251 & 578 & 555 & 118 & 1236 & 607 & 514 & 115 & 1711 & 791 & 1728 & 744 & 0.316 & 0.681 \\
\hline Picelli 2010 [22] & 1781 & 819 & 781 & 181 & 1701 & 832 & 708 & 161 & 2419 & 1143 & 2372 & 1030 & 0.321 & 0.560 \\
\hline Shi 2010 [12] & 204 & 185 & 17 & 2 & 204 & 192 & 11 & 1 & 387 & 21 & 395 & 13 & 0.051 & 0.072 \\
\hline Campbell 2009 [41] & 1601 & 764 & 678 & 159 & 1944 & 937 & 848 & 159 & 2206 & 996 & 2722 & 1166 & 0.311 & 0.087 \\
\hline Conden 2009 [37] & 287 & 129 & 129 & 29 & 546 & 255 & 251 & 40 & 387 & 187 & 761 & 331 & 0.326 & 0.039 \\
\hline Joshi 2009 [31] & 301 & 161 & / & l & 354 & 194 & / & I & l & 1 & l & / & I & I \\
\hline Nejda 2009 [38] & 140 & 41 & 72 & 27 & 125 & 64 & 44 & 17 & 154 & 126 & 172 & 78 & 0.450 & 0.044 \\
\hline Tanaka 2009 [60] & 177 & 159 & 16 & 2 & 131 & 120 & 11 & 0 & 334 & 20 & 251 & 11 & 0.056 & 0.616 \\
\hline An 2008 [61] & 500 & 479 & 20 & 1 & 504 & 493 & 11 & 0 & 978 & 22 & 997 & 11 & 0.022 & 0.804 \\
\hline Christensen 2008 [23] & 380 & 172 & 170 & 38 & 770 & 364 & 327 & 79 & 514 & 246 & 1055 & 485 & 0.324 & 0.661 \\
\hline Berndt 2007 [24] & 211 & 100 & 94 & 17 & 2090 & 968 & 896 & 226 & 294 & 128 & 2832 & 1348 & 0.303 & 0.387 \\
\hline Raptis 2007 [21] & 929 & 451 & 391 & 87 & 1098 & 514 & 485 & 99 & 1293 & 565 & 1513 & 683 & 0.304 & 0.310 \\
\hline Landi 2006 [65] & 291 & 145 & 123 & 23 & 309 & 129 & 151 & 29 & 413 & 169 & 409 & 209 & 0.290 & 0.107 \\
\hline Mei 2006 [14] & 160 & 144 & 14 & 2 & 150 & 141 & 9 & 0 & 302 & 18 & 291 & 9 & 0.056 & 0.705 \\
\hline Song 2006 [39] & 1022 & 507 & 418 & 97 & 1224 & 624 & 477 & 123 & 1432 & 612 & 1725 & 723 & 0.299 & 0.026 \\
\hline Kim 2004 [68] & 107 & 100 & 7 & 0 & 330 & 311 & 18 & 1 & 207 & 7 & 640 & 20 & 0.033 & 0.192 \\
\hline Listgarten 2004 [40] & 170 & 89 & 64 & 17 & 156 & 76 & 75 & 5 & 242 & 98 & 227 & 85 & 0.288 & 0.008 \\
\hline Mathonnet 2003 [70] & 287 & 149 & 112 & 26 & 320 & 154 & 132 & 34 & 410 & 164 & 440 & 200 & 0.286 & 0.474 \\
\hline \multicolumn{15}{|l|}{$1151 \mathrm{~T}>\mathrm{A}(\mathrm{rs63750447)}$} \\
\hline Peng2016 [1] & 156 & 142 & 13 & 1 & 311 & 310 & 1 & 0 & 297 & 15 & 621 & 1 & 0.048 & 0.977 \\
\hline Shi 2010 [12] & 204 & 178 & 24 & 2 & 204 & 191 & 12 & 1 & 380 & 28 & 394 & 14 & 0.069 & 0.108 \\
\hline Ohsawa 2009 [13] & 670 & 630 & 39 & 1 & 332 & 327 & 5 & 0 & 1299 & 41 & 659 & 5 & 0.031 & 0.890 \\
\hline Mei 2006 [14] & 160 & 142 & 18 & 0 & 150 & 141 & 9 & 0 & 302 & 18 & 291 & 9 & 0.056 & 0.705 \\
\hline Zhang 2004 (EC) [8] & 233 & 206 & 27 & 0 & 268 & 251 & 17 & 0 & 439 & 27 & 519 & 17 & 0.058 & 0.592 \\
\hline Zhang 2005 (CRC) [8] & 90 & 82 & 8 & 0 & 268 & 251 & 17 & 0 & 172 & 8 & 519 & 17 & 0.044 & 0.592 \\
\hline Zhang 2004 (BC) [8] & 111 & 104 & 7 & 0 & 268 & 251 & 17 & 0 & 215 & 7 & 519 & 17 & 0.032 & 0.592 \\
\hline Zhang 2004 (GC) [8] & 273 & 240 & 33 & 0 & 268 & 251 & 17 & 0 & 513 & 33 & 519 & 17 & 0.060 & 0.592 \\
\hline Wang 2000 (CRC) [29] & 101 & 88 & 13 & 0 & 100 & 94 & 6 & 0 & 189 & 13 & 194 & 6 & 0.064 & 0.757 \\
\hline Wang 2000 (EC) [29] & 76 & 69 & 7 & 0 & 100 & 94 & 6 & 0 & 145 & 7 & 194 & 6 & 0.046 & 0.757 \\
\hline Wang 2000 (GC) [29] & 79 & 68 & 11 & 0 & 100 & 94 & 6 & 0 & 147 & 11 & 194 & 6 & 0.070 & 0.757 \\
\hline
\end{tabular}

A: the major allele, B: the minor allele, MAF: minor allele frequencies; HWE: Hardy-Weinberg equilibrium. 


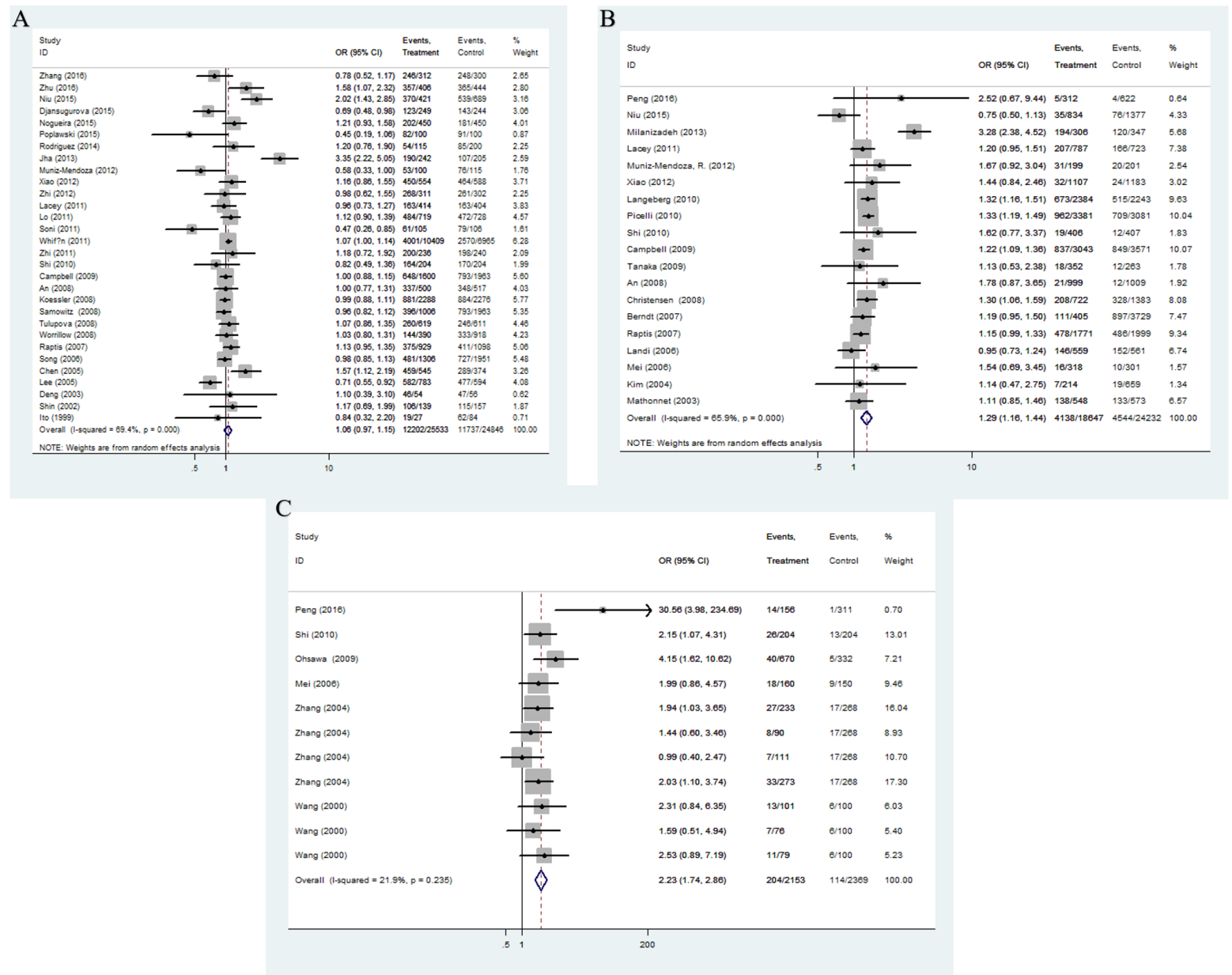

Figure 2: Forest plot of OR with 95\%CI for the hMLH1 polymorphisms with cancer risk under dominate model according to HWE ((A) rs1800734; (B) rs1799977; (C) rs63750447). CI: confidence interval, OR: odds ratio, HWE: Hardy-Weinberg equilibrium.
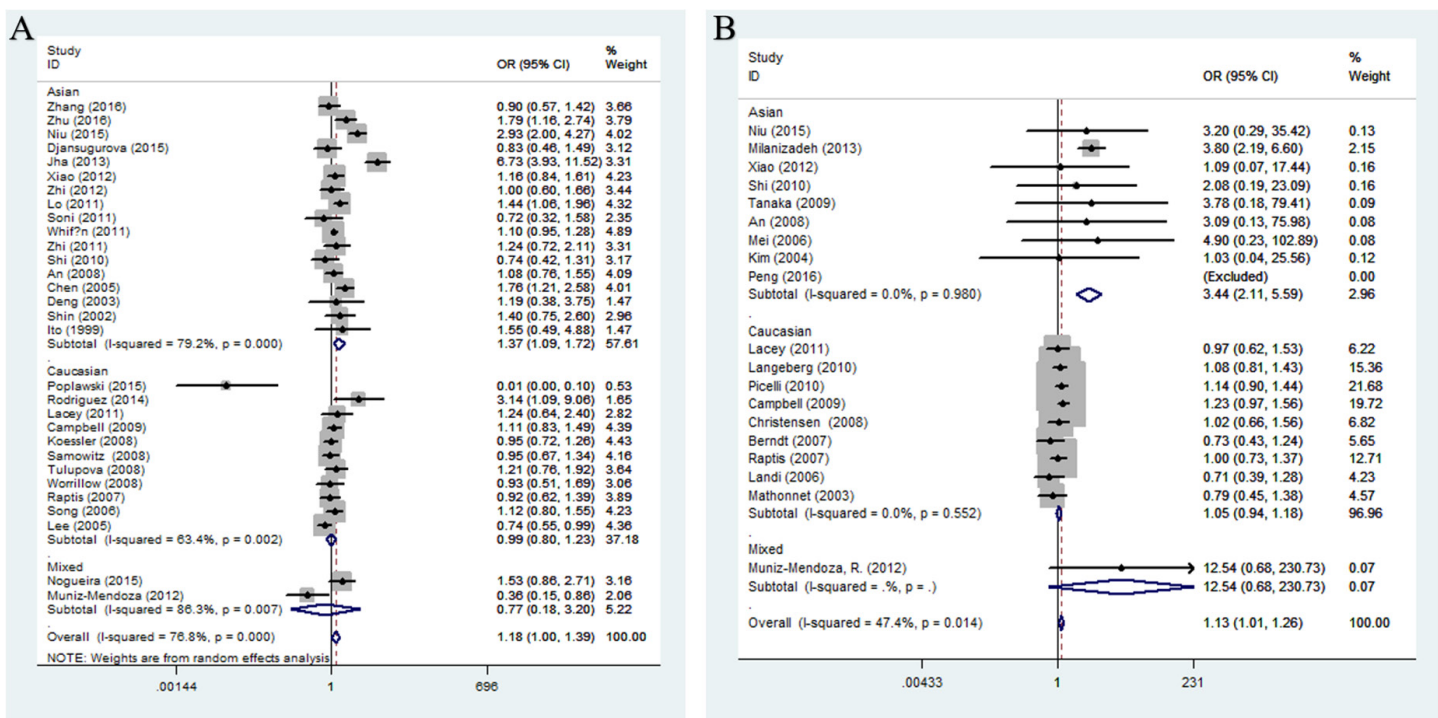

Figure 3: Stratified analysis by ethnicity for the association between hMLH1 polymorphisms and cancer risk under homozygote model according to HWE ((A) rs1800734; (B) rs1799977). CI: confidence interval, OR: odds ratio, HWE: Hardy-Weinberg equilibrium. 
1.03-1.42, $P=0.023)$ and prostate cancer under dominant model $(\mathrm{OR}=1.36,95 \% \mathrm{CI}=1.16-1.59, P<0.0001)$.

Besides, the results of subgroup analyses by source of control and study design exhibited in the Supplementary Table 1.

\section{Rs63750447 polymorphism}

A total of 2153 cancer cases and 1365 cancerfree controls from 11 studies were involved in our meta-analysis for rs63750447 polymorphism. Since the homozygous mutant AA of rs63750447 polymorphism was in very rare frequencies, we chose allele model, heterozygous model and dominant model to evaluate the association strength. The pooled analysis observed a significant association between cancer risk and rs63750447 polymorphism (dominant comparison: OR $=2.23,95 \% \mathrm{CI}=1.75-2.86, P<0.0001$; heterozygote comparison: $\mathrm{OR}=2.21,95 \% \mathrm{CI}=1.73-2.84, P<0.0001$; allele comparison: $\mathrm{OR}=2.19,95 \% \mathrm{CI}=1.72-2.78, P<$ 0.0001 ), as shown in Figure 2C.

The subgroup analysis by cancer type (Figure 4B) indicated that rs63750447 polymorphism had influences on colorectal cancer (dominant comparison: $\mathrm{OR}=2.87,95 \% \mathrm{CI}=1.42-5.82, P=0.003$; heterozygote comparison: $\mathrm{OR}=2.81,95 \% \mathrm{CI}=1.42-5.57, P=0.003$; allele comparison: $\mathrm{OR}=2.84,95 \% \mathrm{CI}=1.38-5.81, P$ $=0.004$ ), gastric cancer (dominant comparison: OR $=2.15,95 \% \mathrm{CI}=1.27-3.64, P=0.005$; heterozygote comparison: $\mathrm{OR}=2.2115,95 \% \mathrm{CI}=1.27-3.64, P=0.005$; allele comparison: $\mathrm{OR}=2.19,95 \% \mathrm{CI}=1.24-3.47, P=$ 0.006), and endometrium cancer (dominant comparison: $\mathrm{OR}=2.23,95 \% \mathrm{CI}=1.06-3.21, P=0.029$; heterozygote comparison: $\mathrm{OR}=1.85,95 \% \mathrm{CI}=1.06-3.21, P=0.029$; allele comparison: $\mathrm{OR}=1.80,95 \% \mathrm{CI}=1.05-3.09, P=$ $0.033)$. When we conducted the subgroup analysis by quality score, there was a significantly increased cancer risk for rs63750447 polymorphism in both high-quality studies and low-quality studies (shown in Supplementary Table 1).

\section{Test of heterogeneity and sensitivity analysis}

As shown in Supplementary Table 1, significant heterogeneities existed after pooled the data of rs 1800734 and rs1799977 polymorphisms under different comparison models $\left(P \leq 0.10\right.$ or $\mathrm{I}^{2} \geq 50 \%$ ), thus further subgroup analyses base on ethnicity, cancer type, source of control, and quality scores were performed. No obvious heterogeneity was found for rs63750447 polymorphism $\left(P>0.10\right.$ or $\left.\mathrm{I}^{2}<50 \%\right)$. Subsequent sensitivity analysis proved the stability of our study, since no significant alteration was detected after removing each individual study and rechecking the pooled ORs and 95\%CIs for the rs1800734 and rs1799977 polymorphisms (Figure 5A, 5B). The third study performed by Zhang et al seemingly altered the pooled ORs significantly (Figure 5), and the detailed data from Stata 14.0 also showed us it was nearly approached to the upper limit. We guess it was due to the sample size of rs63750447 polymorphism was insufficient, only 11 studies from 6 articles were included. It indicated us the overall results of rs63750447 should be treated more carefully.

\section{Publication bias}

The possible publication bias in the eligible literature was evaluated by Egger's test and funnel plots. As shown in Figure 6, the Begg's funnel plots appear to be symmetrical. This symmetry was then confirmed by the statistical results of Egger's test $(P>0.05$, shown in Table 3 ). These provided evidence for the absence of publication bias.

\section{DISCUSSION}

To elucidate the veritable relationship between three hMLH1 polymorphisms (rs1800734; rs1799977; rs63750447) and cancer risk, we performed this metaanalysis based on overall published data up to May 2017. We found all of these polymorphisms can enhance overall cancer risks, especially Asians, under different genetic comparisons (Supplementary Table 1). Further subgroup analyses were carried out according to cancer type, source of control, quality score, and study design, and results worth discussing were obtained.

Interestingly, we found a moderate association existing between rs 1800734 and the risk of gastric cancer in three genetic models $(\mathrm{OR}=1.14, P=0.017$; $\mathrm{OR}=$ 1.33, $P=0.019 ; \mathrm{OR}=1.27, P=0.024)$ and lung cancer in recessive model $(\mathrm{OR}=1.27, P=0.024)$, while no connection was display with colorectal cancer. As far as we know now, microsatellite instability (MSI) often occurs when mismatch errors failed to be corrected or hMLH1 gene was epigenetic silencing. Campbell et al. [41] found rs1800734 polymorphism enhanced MSI-positive colorectal cancer, the association was proved by Mrkonjic et al. [42] due to the effects of rs1800734 on the MLH1 promoter methylation, immunohistochemistry (IHC) deficiency, or both. This indicated us when performing further studies focused on the relationship between rs1800734 and cancer risk, the MSI-statue of cancer patients should be evaluated fundamentally.

Rs1799977 was a nonsynonymous coding polymorphism in hMLH1, which leaded to an amino acid change from isoleucine to valine. The mutational $\mathrm{G}$ allele of rs1799977 polymorphism was proved to connect with susceptibility of colorectal cancer and prostate cancer. For rs63750447, the cancer-specific analysis showed an increased risk of colorectal cancer, endometrial cancer and gastric cancer. Recently, rs63750447 was observed overexpressed in patients with EGFR-TKI (epidermal growth 
A

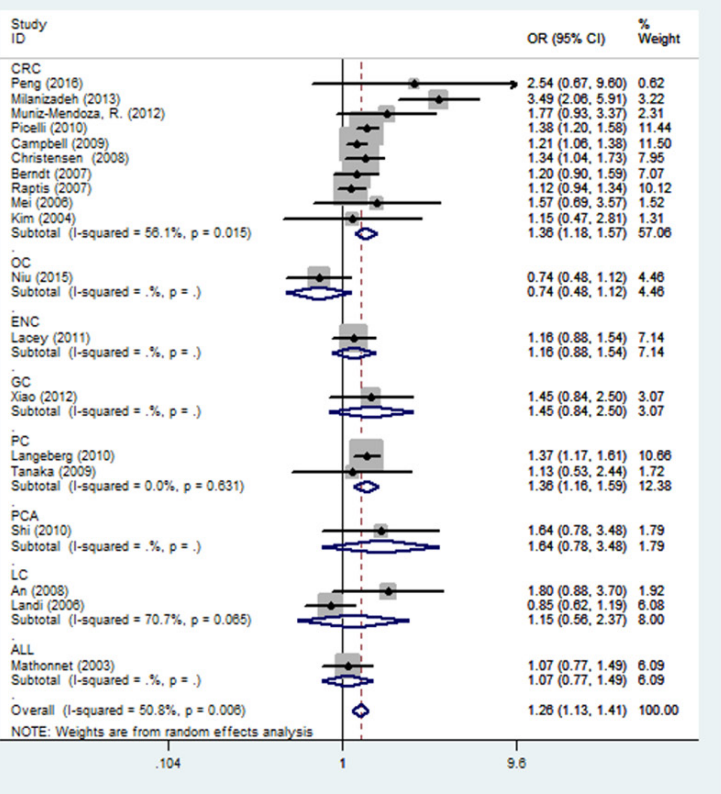

B

\begin{tabular}{|c|c|c|c|}
\hline \multicolumn{2}{|l|}{$\begin{array}{l}\text { Study } \\
\text { ID }\end{array}$} & OR $(95 \% \mathrm{Cl})$ & $\begin{array}{l}\% \\
\text { Weight }\end{array}$ \\
\hline \multirow{2}{*}{\multicolumn{4}{|c|}{$\begin{array}{l}\text { CRC } \\
\text { Peng (2016) }\end{array}$}} \\
\hline & & $30.56(3.98,234.69$ & 0.70 \\
\hline Ohsawa (2009) & \pm & $4.15(1.62,10.62)$ & 7.21 \\
\hline Mei (2006) & $\rightarrow$ & $1.99(0.86,4.57)$ & 9.46 \\
\hline Zhang (2004) & & $1.44(0.60,3.46)$ & 8.93 \\
\hline Wang (2000) & - & $2.31(0.84,6.35)$ & 6.03 \\
\hline Subtotal $(1-$ squared $=57.1 \%, p=0.054)$ & 3 & $3.00(1.97,4.55)$ & 32.33 \\
\hline \multicolumn{4}{|l|}{ TC } \\
\hline Shi (2010) & & $2.15(1.07,4.31)$ & 13.01 \\
\hline Subtotal $(1-$ squared $=. \%, p=)$. & & $2.15(1.07,4.31)$ & 13.01 \\
\hline \multicolumn{4}{|l|}{ EC } \\
\hline Zhang (2004) & & $1.94(1.03,3.65)$ & 16.04 \\
\hline Wang (2000) & & $1.59(0.51,4.94)$ & 5.40 \\
\hline Subtotal (I-squared $=0.0 \%, p=0.766)$ & & $1.85(1.06,3.21)$ & 21.43 \\
\hline \multicolumn{4}{|l|}{$B C$} \\
\hline Zhang (2004) & 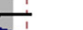 & $0.99(0.40,2.47)$ & 10.70 \\
\hline Subtotal (I-squared $=. \%, p=$.) & & $0.99(0.40,2.47)$ & 10.70 \\
\hline \multicolumn{4}{|l|}{ GC } \\
\hline Zhang (2004) & - & $2.03(1.10,3.74)$ & 17.30 \\
\hline Wang (2000) & & $2.53(0.89,7.19)$ & 5.23 \\
\hline Subtotal (I-squared $=0.0 \%, p=0.719$ ) & & $2.15(1.27,3.64)$ & 22.53 \\
\hline Overall (I-squared $=21.9 \%, p=0.235)$ & $\infty$ & $2.23(1.74,2.86)$ & 100.00 \\
\hline .00426 & & & \\
\hline
\end{tabular}

Figure 4: Stratified analysis by cancer type for the association between hMLH1 polymorphisms and cancer risk under dominant model according to HWE ((A) rs1799977; (B) rs63750447). CI: confidence interval, OR: odds ratio. CRC: colorectal cancer; GC: gastric cancer; BC: breast cancer; PC: prostate cancer; EC: endometrial cancer; OC: ovarian carcinoma; GC: gastric cancer; LC: lung cancer; other: other cancer; HWE: Hardy-Weinberg equilibrium.

A

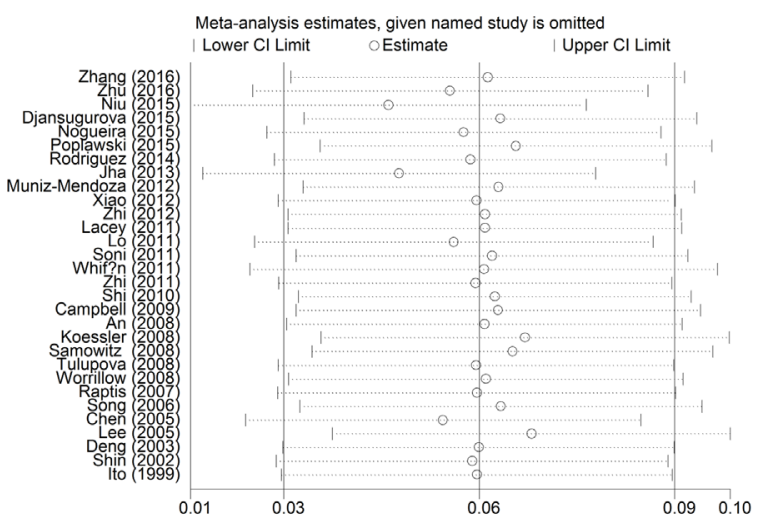

B

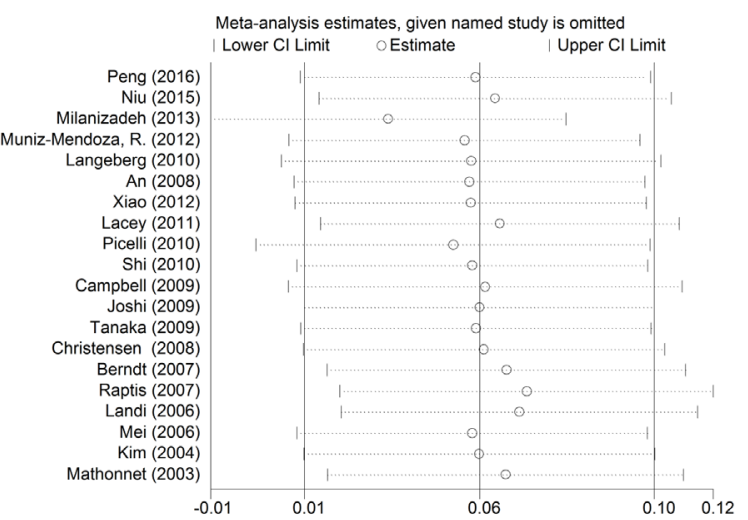

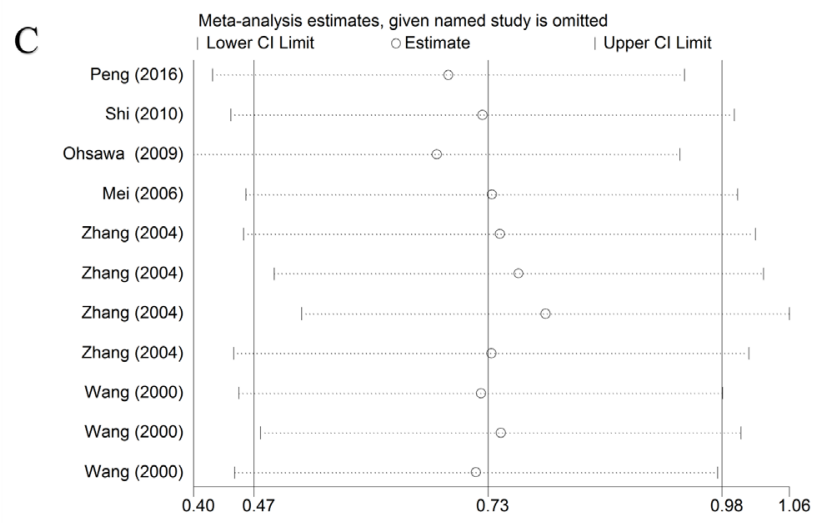

Figure 5: Sensitivity analysis of the associations between hMLH1 polymorphisms and cancer risk according to HWE ((A) rs1800734; (B) rs1799977; (C) rs63750447). HWE: Hardy-Weinberg equilibrium. 
Table 3: Egger's test for publication bias test of hMLH1 polymorphisms

\begin{tabular}{lcccccc}
\hline Egger's test & SE & Coef & Std. Err & $\mathbf{t}$ & $\mathbf{P}>|\mathbf{t}|$ & $\mathbf{9 5 \% C I}$ \\
\hline rs1800734 & slope & 0.06249 & 0.064308 & 0.97 & 0.337 & {$[-0.067807,0.192794]$} \\
rs1799977 & bias & 0.15166 & 0.749679 & 0.20 & 0.841 & {$[-1.367335,1.670654]$} \\
& slope & 0.17888 & 0.082661 & 2.16 & 0.042 & {$[0.007456,0.350311]$} \\
rs63750447 & bias & 0.48454 & 0.597343 & 0.81 & 0.426 & {$[-0.754272,1.723357]$} \\
& slope & -0.12387 & 0.497384 & -0.25 & 0.809 & {$[-1.249034,1.001287]$} \\
& bias & 2.03105 & 1.146982 & 1.77 & 0.110 & {$[-0.563603,4.625704]$} \\
\hline
\end{tabular}

SE: standard error; 95\%CI: 95\% confidence interval.

factor receptor-tyrosine kinase inhibitor) resistance, which has a possible shorter progression-free survival [43]. Thus, it was speculated that MLH1 might be involved in EGFR signaling or other pathways (such as proliferation and survival) [1].

Compare with previous meta-analyses study on the association between hMLH1 and cancer risk, our study included a larger sample size and performed more detailed stratification analysis. Besides, our study has stricter inclusion criteria and exclude criteria, thus avoided omissive and false drop (refer to the section of Characteristics of eligible studies, paragraph one). Thus, we think our results are more reliable and convinced.
Moreover, we found rs1800734 was related to gastric cancer, while rs1799977 may have an influence on colorectal and prostate cancer. It may give us some hints for the further study.

There are still some limitations existing in this metaanalysis. Firstly, insufficiency of original data limited us to proceed more accurate analyses on the potential interaction between these polymorphisms and other risk factors such as age, sex, hereditary background, lifestyle, and MSI status, etc. Secondly, the studies involved in the rs63750447 analysis was insufficient, whose statistical significance was needed to verify by further well-designed study with larger sample sizes. Thirdly, we couldn't


Figure 6: Funnel plots of publication bias ((A) rs1800734; (B) rs1799977; (C) rs63750447). 
exclude the publication bias absolutely according to the negative results of Egger's test and funnel plots. Fourthly, the sample size was still small for any given cancer type, although we have pooled all published literatures. Hence, all the three hMLH1 polymorphisms were associated with cancer risk, but further profoundly investigation was requisite to clarify the strength of these associations.

\section{MATERIALS AND METHODS}

PRISMA statement was used to guide the process of this meta-analysis [44].

\section{Search strategy}

A comprehensive literature search was conducted using the following search terms: ("cancer", "carcinoma", "tumor", "tumour", or "neoplasm") and ("polymorphism", "variation", "variant", or "mutation") and ("hMLH1"). The PubMed, Web of knowledge, VIP, WanFang and Chinese National Knowledge Infrastructure (CNKI) databases were searched up to May, 2017. Additional studies were identified by manual search of the references of the original studies or review articles. This study was approved by the ethics committee of Xi'an Jiaotong University.

To be eligible for this meta-analysis, the included study was required to (1) be case-control or case-cohort studies; (2) focused on the relationship between hMLH1 polymorphisms and risk of any cancer; (3) have at least three articles for each studied hMLH1 polymorphism, and available information concerning the genotype frequency of each included SNP of hMLH1 (i.e., rs1800734; rs 1799977; rs63750447); (4) be published in English or Chinese. The exclusion criteria were as follows: (1) studies were not focused on cancer risk or targeted hMLH1 SNPs (rs1800734; 2: rs1799977; 3: rs63750447); (2) studies failed to supply any data on genotype distribution, (3) studies were updated by a following study where a larger number of subjects were included, (4) studies were designed as a case-case or case-only study. If 2 or more studies contained overlapping data, we selected the paper included more samples. Studies containing two or more case-control groups were considered as two or more independent studies.

\section{Data extraction and quality assessment}

For each included study, two investigators independently extracted the raw data and demographic information, including publication year, first author, ethnicity and country or origin, the number of cases and controls, source of controls, genotyping methods, genetic distribution, and $\mathrm{P}$ value of Hardy-Weinberg equilibrium (HWE) among the controls. Studies not follow HWE were excluded in subgroup analysis. We applied the Newcastle-
Ottawa Scale (NOS) to evaluate the methodological quality of the eligible studies according to Zeng et al [45]. Accumulated score ranges from 0 to 9 points, and a score of 0-5 and 6-9 is considered to suggest a low and high quality respectively, with higher quality representing lower risks of bias. A discussion or consultation with a senior author was conducted to settle controversy until a consensus was reached.

\section{Statistical analysis}

To evaluate the strength of association between hMLH1 polymorphisms (rs1800734; rs1799977; rs63750447) and cancer risk, we calculated the odds ratios (ORs) and 95\% confidence intervals (CIs) based on the genotype and allele frequencies in cases and controls of each eligible study. We used the $\mathrm{Z}$ test to access the significance of all pooled ORs and it was considered statistically significant if the $\mathrm{P}$ value $<0.05$. The Chisquare-based Q statistic test and $\mathrm{I}^{2}$ statistic were applied to examine the statistical heterogeneity among studies. When no obvious heterogeneity existed across the studies ( $\mathrm{P}>0.10$ or $\left.\mathrm{I}^{2}<50 \%\right)$, we pooled the ORs using fixed-effect model (Mantel- Haenszel); otherwise, the random effects model (DerSimonian and Laird) was chosen. The potential publication bias was evaluated by funnel plot and Egger's test. To access the stability of the results in this meta-analysis, we performed sensitivity analysis by sequentially excluding each study and rechecked whether the pooled ORs were altered significantly.

The following genetic models were evaluated: allele comparison (B vs. A), homozygote comparison (BB vs. $\mathrm{AA}$ ), heterozygote comparison ( $\mathrm{AB}$ vs. $\mathrm{AA}$ ), recessive model (BB vs. $\mathrm{AA}+\mathrm{AB})$, and dominant model $(\mathrm{BB}+$ $A B$ vs. AA). "A" represents the wild allele, while " $B$ " represents the mutation allele. After excluded studies not according to HWE, we conducted the subgroup analysis based on ethnicity (divided into Asian and Caucasian), cancer type, and source of control. All statistical analyses were calculated with the software STATA (Version 14.0; Stata Corp, College Station, TX).

\section{CONFLICTS OF INTEREST}

We declare that we have no conflicts of interest.

\section{FUNDING}

This work was supported by the National Social Science Foundation of China (16BGL183), the Natural Science Foundation of Shaanxi Province (2015JM8415) and the Fundamental Research Funds for the Central Universities of China (2011jdhz55). 


\section{REFERENCES}

1. Peng $\mathrm{HX}, \mathrm{Xu} \mathrm{X}$, Yang $\mathrm{R}$, Chu YM, Yang DM, Xu Y, Zhou FL, Ma WZ, Zhang XJ, Guan M, Yang ZH, Jin ZD. Molecular analysis of MLH1 variants in Chinese sporadic colorectal cancer patients. Genet Mol Res. 2016.

2. Zhu H, Li X, Zhang X, Chen D, Li D, Ren J, Gu H, Shu Y, Wang D. Polymorphisms in mismatch repair genes are associated with risk and microsatellite instability of gastric cancer, and interact with life exposures. Gene. 2016; 579:52-57.

3. Poplawski T, Sobczuk A, Sarnik J, Pawlowska E, Blasiak J. Polymorphism of DNA mismatch repair genes in endometrial cancer. Exp Oncol. 2015; 37:44-47.

4. Li GM. Mechanisms and functions of DNA mismatch repair. Cell Res. 2008; 18:85-98.

5. Wang Y, Cortez D, Yazdi P, Neff N, Elledge SJ, Qin J. BASC, a super complex of BRCA1-associated proteins involved in the recognition and repair of aberrant DNA structures. Genes Dev. 2000; 14:927-939.

6. Duckett DR, Bronstein SM, Taya Y, Modrich P. hMutSalpha- and hMutLalpha-dependent phosphorylation of p53 in response to DNA methylator damage. Proc Natl Acad Sci U S A. 1999; 96:12384-12388.

7. Ma G, Ge Y, Gu D, Du M, Chu H, Chen J, Zhang Z, Wang M. Functional annotation of colorectal cancer susceptibility loci identifies MLH1 rs1800734 associated with MSI patients. Gut. 2016; 65:1227-1228.

8. Zhang XM, Li JT, Zhu M, Wu XL, Gao P, Zhou P, Wang YP. [Study on the relationship between genetic polymorphism Val384Asp in hMLH1 gene and the risk of four different carcinomas]. [Article in Chinese]. Zhonghua Liu Xing Bing Xue Za Zhi. 2004; 25:978-981.

9. Nizam ZM, Abdul Aziz AA, Kaur G, Abu Hassan MR, Mohd Sidek AS, Lee YY, Mazuwin M, Ankathil R. Contribution of the MLH1 $-93 \mathrm{G}>$ a promoter polymorphism in modulating susceptibility risk in Malaysian colorectal cancer patients. Asian Pac J Cancer Prev. 2013; 14:619-624.

10. Zhang LL, Tang XJ, Wang XY, Zhu YW, Peng XB, Gong L. A promoter polymorphism in the hMLH1 gene (-93G/A) associated with sporadic colorectal cancer. Oncol Lett. 2016; 12:4035-4040

11. Milanizadeh S, Khanyaghma M, Haghighi MM, Mohebbi S, Damavand B, Almasi S, Azimzadeh P, Zali M. Molecular analysis of imperative polymorphisms of MLH1 gene in sporadic colorectal cancer. Cancer Biomark. 2013; 13:427-432.

12. Shi WP, Bian JC, Jiang F, Ni HX, Zhu QX, Tang HW, Shen Q, Wu Y. [Study on genetic polymorphism of human mismatch repair gene hMLH1 and susceptibility of papillary thyroid carcinoma in Chinese Han people]. [Article in Chinese]. Zhonghua Yu Fang Yi Xue Za Zhi. 2010; 44:235-241.
13. Ohsawa T, Sahara T, Muramatsu S, Nishimura Y, Yathuoka T, Tanaka Y, Yamaguchi K, Ishida H, Akagi K. Colorectal cancer susceptibility associated with the hMLH1 V384D variant. Mol Med Rep. 2009; 2:887-891.

14. Mei Q, Yan HL, Ding FX, Xue G, Huang JJ, Wang YZ, Sun SH. Single-nucleotide polymorphisms of mismatch repair genes in healthy Chinese individuals and sporadic colorectal cancer patients. Cancer Genet Cytogenet. 2006; 171:17-23.

15. Chen H, Taylor NP, Sotamaa KM, Mutch DG, Powell MA, Schmidt AP, Feng S, Hampel HL, de la Chapelle A, Goodfellow PJ. Evidence for heritable predisposition to epigenetic silencing of MLH1. Int J Cancer. 2007; 120:1684-1688.

16. Pan XM, Yang WZ, Xu GH, Bai P, Qin HJ, Zhang LS, Zhai XD, Tang M, Deng W, Zhang L, Gao LB. The association between MLH1 -93 G>A polymorphism of DNA mismatch repair and cancer susceptibility: a meta-analysis. Mutagenesis. 2011; 26:667-673.

17. Chen H, Shen Z, Hu Y, Xiao Q, Bei D, Shen X, Ding K. Association between MutL homolog 1 polymorphisms and the risk of colorectal cancer: a meta-analysis. J Cancer Res Clin Oncol. 2015; 141:2147-2158.

18. van Roon EH, van Puijenbroek M, Middeldorp A, van Eijk R, de Meijer EJ, Erasmus D, Wouters KA, van Engeland M, Oosting J, Hes FJ, Tops CM, van Wezel T, Boer JM, Morreau H. Early onset MSI-H colon cancer with MLH1 promoter methylation, is there a genetic predisposition? BMC Cancer. 2010; 10:180.

19. Xu JL, Yin ZQ, Huang MD, Wang XF, Gao W, Liu LX, Wang RS, Huang PW, Yin YM, Liu P, Shu YQ. MLH1 polymorphisms and cancer risk: a meta-analysis based on 33 case-control studies. Asian Pac J Cancer Prev. 2012; 13:901-907.

20. Samowitz WS, Curtin K, Wolff RK, Albertsen H, Sweeney C, Caan BJ, Ulrich CM, Potter JD, Slattery ML. The MLH1 -93 G>A promoter polymorphism and genetic and epigenetic alterations in colon cancer. Genes Chromosomes Cancer. 2008; 47:835-844.

21. Raptis S, Mrkonjic M, Green RC, Pethe VV, Monga N, Chan YM, Daftary D, Dicks E, Younghusband BH, Parfrey PS, Gallinger SS, McLaughlin JR, Knight JA, Bapat B. MLH1 -93G $>$ A promoter polymorphism and the risk of microsatellite-unstable colorectal cancer. J Natl Cancer Inst. 2007; 99:463-474.

22. Picelli S, Zajac P, Zhou XL, Edler D, Lenander C, Dalen J, Hjern F, Lundqvist N, Lindforss U, Pahlman L, Smedh $\mathrm{K}$, Tornqvist A, Holm J, et al. Common variants in human CRC genes as low-risk alleles. Eur J Cancer. 2010; 46:1041-1048.

23. Christensen LL, Madsen BE, Wikman FP, Wiuf C, Koed K, Tjonneland A, Olsen A, Syvanen AC, Andersen CL, Orntoft TF. The association between genetic variants in hMLH1 and hMSH2 and the development of sporadic colorectal cancer in the Danish population. BMC Med Genet. 2008; 9:52. 
24. Berndt SI, Platz EA, Fallin MD, Thuita LW, Hoffman SC, Helzlsouer KJ. Mismatch repair polymorphisms and the risk of colorectal cancer. Int J Cancer. 2007; 120:1548-1554.

25. Martinez-Uruena N, Macias L, Perez-Cabornero L, Infante M, Lastra E, Cruz JJ, Miner C, Gonzalez R, Duran M. Incidence of -93 MLH1 promoter polymorphism in familial and sporadic colorectal cancer. Colorectal Dis. 2013; 15:e118-123.

26. Harley I, Rosen B, Risch HA, Siminovitch K, Beiner ME, McLaughlin J, Sun P, Narod SA. Ovarian cancer risk is associated with a common variant in the promoter sequence of the mismatch repair gene MLH1. Gynecol Oncol. 2008; 109:384-387.

27. Shin KH, Shin JH, Kim JH, Park JG. Mutational analysis of promoters of mismatch repair genes hMSH2 and hMLH1 in hereditary nonpolyposis colorectal cancer and early onset colorectal cancer patients: identification of three novel germ-line mutations in promoter of the hMSH2 gene. Cancer Res. 2002; 62:38-42.

28. Ito E, Yanagisawa Y, Iwahashi Y, Suzuki Y, Nagasaki H, Akiyama Y, Sugano S, Yuasa Y, Maruyama K. A core promoter and a frequent single-nucleotide polymorphism of the mismatch repair gene hMLH1. BiochemBiophys Res Commun. 1999; 256:488-494.

29. Wang Y, Zhou J, Li Z, Wang J, Li J, Gao C, Gao P. [One of the etiological factors of digestive tract cancers in Chinese: the missense mutation Val384Asp in the hMLH1 gene]. [Article in Chinese]. Zhonghua Yi Xue Yi Chuan Xue Za Zhi. 2000; 17:82-86.

30. Muniz-Mendoza R, Ayala-Madrigal ML, Partida-Perez M, Peregrina-Sandoval J, Leal-Ugarte E, Macias-Gomez N, Peralta-Leal V, Meza-Espinoza JP, Moreno-Ortiz JM, Ramirez-Ramirez R, Suarez-Villanueva S, GutierrezAngulo M. MLH1 and XRCC1 polymorphisms in Mexican patients with colorectal cancer. Genet Mol Res. 2012; 11:2315-2320.

31. Joshi AD, Corral R, Siegmund KD, Haile RW, Le Marchand L, Martinez ME, Ahnen DJ, Sandler RS, Lance P, Stern MC. Red meat and poultry intake, polymorphisms in the nucleotide excision repair and mismatch repair pathways and colorectal cancer risk. Carcinogenesis. 2009; 30:472-479.

32. Savio AJ, Lemire M, Mrkonjic M, Gallinger S, Zanke BW, Hudson TJ, Bapat B. MLH1 region polymorphisms show a significant association with $\mathrm{CpG}$ island shore methylation in a large cohort of healthy individuals. PLoS One. 2012; 7:e51531.

33. Shih CM, Chen CY, Lee IH, Kao WT, Wang YC. A polymorphism in the hMLH1 gene $(-93 \mathrm{G} \curvearrowright \mathrm{A})$ associated with lung cancer susceptibility and prognosis. Int J Mol Med. 2009.

34. Scott K, Adamson PJ, Willett EV, Worrillow LJ, Allan JM. Genetic variation in genes expressed in the germinal center and risk of B cell lymphoma among Caucasians. Haematologica. 2008; 93:1597-1600.
35. Beiner ME, Rosen B, Fyles A, Harley I, Pal T, Siminovitch K, Zhang S, Sun P, Narod SA. Endometrial cancer risk is associated with variants of the mismatch repair genes MLH1 and MSH2. Cancer Epidemiol Biomarkers Prev. 2006; 15:1636-1640.

36. Park SH, Lee GY, Jeon HS, Lee SJ, Kim KM, Jang SS, Kim CH, Lee WK, Kam S, Park RW, Kim IS, Jung TH, Park JY. -93G-->A polymorphism of hMLH1 and risk of primary lung cancer. Int J Cancer. 2004; 112:678-682.

37. Conde J, Silva SN, Azevedo AP, Teixeira V, Pina JE, Rueff J, Gaspar JF. Association of common variants in mismatch repair genes and breast cancer susceptibility: a multigene study. BMC Cancer. 2009; 9:344.

38. Nejda N, Iglesias D, Moreno Azcoita M, Medina Arana V, Gonzalez-Aguilera JJ, Fernandez-Peralta AM. A MLH1 polymorphism that increases cancer risk is associated with better outcome in sporadic colorectal cancer. Cancer Genet Cytogenet. 2009; 193:71-77.

39. Song H, Ramus SJ, Quaye L, DiCioccio RA, Tyrer J, Lomas E, Shadforth D, Hogdall E, Hogdall C, McGuire V, Whittemore AS, Easton DF, Ponder BA, et al. Common variants in mismatch repair genes and risk of invasive ovarian cancer. Carcinogenesis. 2006; 27:2235-2242.

40. Listgarten J, Damaraju S, Poulin B, Cook L, Dufour J, Driga A, Mackey J, Wishart D, Greiner R, Zanke B. Predictive models for breast cancer susceptibility from multiple single nucleotide polymorphisms. Clin Cancer Res. 2004; 10:2725-2737.

41. Campbell PT, Curtin K, Ulrich CM, Samowitz WS, Bigler J, Velicer CM, Caan B, Potter JD, Slattery ML. Mismatch repair polymorphisms and risk of colon cancer, tumour microsatellite instability and interactions with lifestyle factors. Gut. 2009; 58:661-667.

42. Mrkonjic M, Roslin NM, Greenwood CM, Raptis S, Pollett A, Laird PW, Pethe VV, Chiang T, Daftary D, Dicks E, Thibodeau SN, Gallinger S, Parfrey PS, et al. Specific variants in the MLH1 gene region may drive DNA methylation, loss of protein expression, and MSI-H colorectal cancer. PLoS One. 2010; 5:e13314.

43. Chiu CH, Ho HL, Doong H, Yeh YC, Chen MY, Chou TY, Tsai CM. MLH1 V384D polymorphism associates with poor response to EGFR tyrosine kinase inhibitors in patients with EGFR L858R-positive lung adenocarcinoma. Oncotarget. 2015; 6:8407-8417. https://doi.org/10.18632/ oncotarget. 3511.

44. Moher D, Liberati A, Tetzlaff J, Altman DG, PRISMA Group. Preferred reporting items for systematic reviews and meta-analyses: the PRISMA statement. J Clin Epidemiol. 2009; 62:1006-1012.

45. Zeng X, Zhang Y, Kwong JS, Zhang C, Li S, Sun F, Niu $\mathrm{Y}, \mathrm{Du} \mathrm{L}$. The methodological quality assessment tools for preclinical and clinical studies, systematic review and meta-analysis, and clinical practice guideline: a systematic review. J Evid Based Med. 2015; 8:2-10. 
46. Djansugurova L, Zhunussova G, Khussainova E, Iksan O, Afonin G, Kaidarova D, Parker MI. Association of DCC, MLH1, GSTT1, GSTM1, and TP53 gene polymorphisms with colorectal cancer in Kazakhstan. Tumour Biol. 2015; $36: 279-289$

47. Niu L, Li S, Liang $\mathrm{H}$, Li $\mathrm{H}$. The hMLH1 -93G $>$ A polymorphism and risk of ovarian cancer in the Chinese population. PLoS One. 2015; 10:e0135822.

48. Nogueira GA, Lourenco GJ, Oliveira CB, Marson FA, Lopes-Aguiar L, Costa EF, Lima TR, Liutti VT, Leal F, Santos VC, Rinck-Junior JA, Lima CS. Association between genetic polymorphisms in DNA mismatch repair-related genes with risk and prognosis of head and neck squamous cell carcinoma. Int J Cancer. 2015; 137:810-818.

49. Slovakova P, Majerova L, Matakova T, Skerenova M, Kavcova E, Halasova E. Mismatch repair gene polymorphisms and association with lung cancer development. Adv Exp Med Biol. 2015; 833:15-22.

50. Rodriguez-Hernandez I, Perdomo S, Santos-Briz A, Garcia JL, Gomez-Moreta JA, Cruz JJ, Gonzalez-Sarmiento R. Analysis of DNA repair gene polymorphisms in glioblastoma. Gene. 2014; 536:79-83.

51. Jha R, Gaur P, Sharma SC, Das SN. Single nucleotide polymorphism in hMLH1 promoter and risk of tobaccorelated oral carcinoma in high-risk Asian Indians. Gene. 2013; 526:223-227.

52. Xiao XQ, Gong WD, Wang SZ, Zhang ZD, Rui XP, Wu GZ, Ren F. Polymorphisms of mismatch repair gene hMLH1 and hMSH2 and risk of gastric cancer in a Chinese population. Oncol Lett. 2012; 3:591-598.

53. Zhi Y, Yu J, Liu Y, Wei Q, Yuan F, Zhou X, Song B, Chen Z, Yang J. Interaction between polymorphisms of DNA repair genes significantly modulated bladder cancer risk. Int J Med Sci. 2012; 9:498-505.

54. Lacey JV Jr, Yang H, Gaudet MM, Dunning A, Lissowska J, Sherman ME, Peplonska B, Brinton LA, Healey CS, Ahmed S, Pharoah P, Easton D, Chanock S, GarciaClosas M. Endometrial cancer and genetic variation in PTEN, PIK3CA, AKT1, MLH1, and MSH2 within a population-based case-control study. Gynecol Oncol. 2011; 120:167-173.

55. Lo YL, Hsiao CF, Jou YS, Chang GC, Tsai YH, Su WC, Chen KY, Chen YM, Huang MS, Hsieh WS, Chen CJ, Hsiung CA. Polymorphisms of MLH1 and MSH2 genes and the risk of lung cancer among never smokers. Lung Cancer. 2011; 72:280-286.

56. Soni A, Bansal A, Singh LC, Mishra AK, Majumdar M, Regina T, Mohanty NK, Saxena S. Gene expression profile and mutational analysis of DNA mismatch repair genes in carcinoma prostate in Indian population. OMICS. 2011; 15:319-324.

57. Whiffin N, Broderick P, Lubbe SJ, Pittman AM, Penegar S, Chandler I, Houlston RS. MLH1-93G > A is a risk factor for MSI colorectal cancer. Carcinogenesis. 2011; 32:1157-1161.

58. Zhi W, Xue B, Wang L, Xiao N, He Q, Wang Y, Fan Y. The MLH1 2101C>A (Q701K) variant increases the risk of gastric cancer in Chinese males. BMC Gastroenterol. 2011; $11: 133$.

59. Langeberg WJ, Kwon EM, Koopmeiners JS, Ostrander EA, Stanford JL. Population-based study of the association of variants in mismatch repair genes with prostate cancer risk and outcomes. Cancer Epidemiol Biomarkers Prev. 2010; 19:258-264.

60. Tanaka Y, Zaman MS, Majid S, Liu J, Kawakami K, Shiina H, Tokizane T, Dahiya AV, Sen S, Nakajima K. Polymorphisms of MLH1 in benign prostatic hyperplasia and sporadic prostate cancer. Biochem Biophys Res Commun. 2009; 383:440-444.

61. An Y, Jin G, Wang H, Wang Y, Liu H, Li R, Wang H, Qian J, Sun W, Wang Y, Ma H, Miao R, Hu Z, et al. Polymorphisms in hMLH1 and risk of early-onset lung cancer in a southeast Chinese population. Lung Cancer. 2008; 59:164-170.

62. Koessler T, Oestergaard MZ, Song H, Tyrer J, Perkins B, Dunning AM, Easton DF, Pharoah PD. Common variants in mismatch repair genes and risk of colorectal cancer. Gut. 2008; 57:1097-1101.

63. Tulupova E, Kumar R, Hanova M, Slyskova J, Pardini B, Polakova V, Naccarati A, Vodickova L, Novotny J, Halamkova J, Hemminki K, Vodicka P. Do polymorphisms and haplotypes of mismatch repair genes modulate risk of sporadic colorectal cancer? Mutat Resch. 2008; 648:40-45.

64. Worrillow LJ, Smith AG, Scott K, Andersson M, Ashcroft AJ, Dores GM, Glimelius B, Holowaty E, Jackson GH, Jones GL, Lynch CF, Morgan G, Pukkala E, et al. Polymorphic MLH1 and risk of cancer after methylating chemotherapy for Hodgkin lymphoma. J Med Genet. 2008; 45:142-146.

65. Landi S, Gemignani F, Canzian F, Gaborieau V, Barale R, Landi D, Szeszenia-Dabrowska N, Zaridze D, Lissowska J, Rudnai P, Fabianova E, Mates D, Foretova L, et al. DNA repair and cell cycle control genes and the risk of youngonset lung cancer. Cancer Res. 2006; 66:11062-11069.

66. Chen CC, Yang SY, Liu CJ, Lin CL, Liaw YF, Lin SM, Lee SD, Chen PJ, Chen CJ, Yu MW. Association of cytokine and DNA repair gene polymorphisms with hepatitis B-related hepatocellular carcinoma. IntJ Epidemiol. 2005; 34:1310-1318.

67. Lee KM, Choi JY, Kang C, Kang CP, Park SK, Cho H, Cho DY, Yoo KY, Noh DY, Ahn SH, Park CG, Wei Q, Kang D. Genetic polymorphisms of selected DNA repair genes, estrogen and progesterone receptor status, and breast cancer risk. Clin Cancer Res. 2005; 11:4620-4626.

68. Kim JC, Roh SA, Koo KH, Ka IH, Kim HC, Yu CS, Lee KH, Kim JS, Lee HI, Bodmer WF. Genotyping possible polymorphic variants of human mismatch repair genes in 
healthy Korean individuals and sporadic colorectal cancer patients. Fam Cancer. 2004; 3:129-137.

69. Deng DJ, Zhou J, Zhu BD, Ji JF, Harper JC, Powell SM. Silencing-specific methylation and single nucleotide polymorphism of hMLH1 promoter in gastric carcinomas. World J Gastroenterol. 2003; 9:26-29.
70. Mathonnet G, Krajinovic M, Labuda D, Sinnett D. Role of DNA mismatch repair genetic polymorphisms in the risk of childhood acute lymphoblastic leukaemia. Br J Haematol. 2003; 123:45-48. 